\title{
RESEARCH
}

Open Access

\section{Hyperhomocysteinemia and dyslipidemia in point mutation G307S of cystathionine $\beta$ - synthase-deficient rabbit generated using CRISPR/Cas9}

Ting Zhang ${ }^{1,2}$, Rui Lu ${ }^{3}$, Yibing Chen ${ }^{1,2}$, Yuguo Yuan ${ }^{1,2}$, Shaozheng Song ${ }^{4}$, Kunning Yan ${ }^{5}$, Yiwen Zha ${ }^{5}$, Wenwen Zhuang ${ }^{5}$, Yong Cheng ${ }^{1,2^{*}}$ and Jingyan Liang ${ }^{5^{*}}$

\begin{abstract}
Background: Congenital hyper-homocysteinemia (HHcy) is caused by a defective cystathionine $\beta$-synthase (CBS) gene, and is frequently associated with dyslipdemia. The aim of this study was to further elucidate the effect of mutated CBS gene on circulating lipids using a rabbit model harboring a homozygous G307S point mutation in CBS.

Methods: CRISPR/Cas9 system was used to edit the CBS gene in rabbit embryos. The founder rabbits were sequenced, and their plasma homocysteine (Hcy) and lipid profile were analyzed.

Results: Six CBS-knockout (CBS-KO) founder lines with biallelic modifications were obtained. Mutation in CBS caused significant growth retardation and high mortality rates within 6 weeks after birth. In addition, the 6-week old CBS-KO rabbits showed higher plasma levels of Hcy, triglycerides (TG), total cholesterol (TC) and low-density lipoprotein cholesterol (LDL-C) compared to the age-matched wild-type (WT) controls. Histological analysis of the mutants showed accumulation of micro-vesicular cytoplasmic lipid droplets in the hepatocytes. However, gastric infusion of vitamin B and betaine complex significantly decreased the plasma levels of TG, TC and LDL-C in the CBS-KO rabbits, and alleviated hepatic steatosis compared to the untreated animals.
\end{abstract} Conclusion: A CBS ${ }^{\mathrm{G} 3075}$ rabbit model was generated that exhibited severe dyslipidemia when fed on a normal diet, indicating that G307S mutation in the CBS gene is a causative factor for dyslipidemia.

Keywords: Cystathionine $\beta$-synthase, Hyperhomocysteinemia, Dyslipidemia, Rabbits, CRISPR/Cas9, G307S mutation

\footnotetext{
* Correspondence: chengyong@yzu.edu.cn; jyliang@yzu.edu.cn

'College of Veterinary Medicine, Yangzhou University, Yangzhou 225009, Jiangsu, China

${ }^{5}$ Institute of Translational Medicine, Medical College, Yangzhou University, Yangzhou 225001, Jiangsu, China

Full list of author information is available at the end of the article
}

C C The Author(s). 2020 Open Access This article is licensed under a Creative Commons Attribution 4.0 International License, which permits use, sharing, adaptation, distribution and reproduction in any medium or format, as long as you give appropriate credit to the original author(s) and the source, provide a link to the Creative Commons licence, and indicate if changes were made. The images or other third party material in this article are included in the article's Creative Commons licence, unless indicated otherwise in a credit line to the material. If material is not included in the article's Creative Commons licence and your intended use is not permitted by statutory regulation or exceeds the permitted use, you will need to obtain permission directly from the copyright holder. To view a copy of this licence, visit http://creativecommons.org/licenses/by/4.0/. The Creative Commons Public Domain Dedication waiver (http://creativecommons.org/publicdomain/zero/1.0/) applies to the data made available in this article, unless otherwise stated in a credit line to the data. 


\section{Introduction}

Homocysteine (Hcy) is a non-essential sulphur-containing amino acid derived from methionine metabolism. The plasma Hcy range in healthy individuals is $5-15 \mu \mathrm{mol} / \mathrm{L}$, and an increase to $16-30 \mu \mathrm{mol} / \mathrm{L}, \quad 31-100 \mu \mathrm{mol} / \mathrm{L}$ and $>100 \mu \mathrm{mol} / \mathrm{L}$ result in moderate, intermediate and severe hyper-homocysteinemia (HHcy) respectively [1]. Moderate HHcy is present in $5-7 \%$ of the general population, and increases the risk of fatty liver, diabetes, atherosclerosis [2-5], atherothrombosis, stroke, ischemic heart disease and peripheral vascular disease [6-11]. In addition, a $2.5 \mu \mathrm{mol} / \mathrm{L}$ increase in Hcy increases the risk of cardiovascular diseases (CVDs) by $10 \%$ [12], indicating its potential as a biomarker of coronary heart failure $[9,13,14]$.

The causative factors of HHcy include genetics, nutrition, medication, disease status, smoking and age. In addition, severe HHcy is often triggered by congenital deficiency of cystathionine $\beta$-synthase $(C B S)$ or 5,10-methylenetetrahydrofolate reductase (MTHFR) [15], and the most frequent cause is a rare autosomal recessive mutation in the $C B S$ gene. In fact, around 22 mutant alleles of $C B S$ have been reported so far in individuals with $C B S$ deficiency [16-22], and 10 missense mutations including G307S, I278T, V320A, T353M, L101P, A226T, N228S, A231L, D376N and Q526K have been identified by DNA sequencing [23]. The most common mutations are I278T and G307S from exon 8 . One study reported $21 \%$ prevalence of the G307S mutation in UK, $8 \%$ in US patients and $71 \%$ in Ireland [24].

Hcy is re-methylated to methionine by 5methyltetrahydrofolate-homocysteine methyltransferase (MHMT) and the cofactor folate-cobalamin, and the methyl group is donated by betaine via betainehomocysteine methyl transferase (BHMT). In addition, CBS metabolizes Hcy to cystathionine in the presence of vitamin B6 [25-30]. Interestingly, there is considerable clinical heterogeneity among individuals with homocystinuria based on their responsiveness to pyridoxine (vitamin B6) [31]. While patients harboring the G307S mutation are completely non-responsive, those with $\mathrm{I} 278 \mathrm{~T}$ partially respond to B6. In addition, pyridoxine resistance is associated with a more severe clinical phenotype [20]. Although the precise mechanism through which G307S affects CBS function is unclear, there is evidence that it restricts the ability of tyrosine at position 308 to assume the proper conformational state required for the pyridoxial-cystathionine intermediate, eventually inhibiting the catalytic performance [32]. In addition, the pyridoxine non-responsive patients may benefit from betaine supplementation [33], either alone or in combination with vitamin B12, folic acid or a methionine-restricted diet.
HHcy is routinely accompanied by life-threatening vascular complications, and individuals with high circulating levels of Hcy are at a higher risk of CVD and increased 5-year mortality [34]. However, most animal models currently used for cardiovascular research do not accurately simulate the human system [35]. For instance, most disease-causing mutations in humans do not replicate the symptoms in rodents, and their short life-span precludes any investigation of long-term effects. Furthermore, rats, mice, tree shrews and dogs are resistant to atherosclerosis and hypercholesterolemia, unlike primates, hamsters and rabbits [36-39]. The rabbit (Oryctolagus cuniculus) model offers several advantages over rodents, such as greater phylogenetic similarity to primates, adequate amount of blood for plasma biochemical analysis, suitable heart size for studying atherosclerosis in both aorta and coronary arteries [35, 40], and a more diverse genetic background which is conducive to modelling complex diseases and simulating the effects of genetic diversity in the human population [35].

Since most $C B S$ mutations in humans are of the missense type caused by base pair substitutions, $\mathrm{HHcy}$ modeling require gene knock-in as opposed to knock-out. To this end, we generated CBS gene mutant rabbits including point mutation G307S using the CRISPR/Cas9 system in order to gain new insights into the HHcy pathogenesis. In addition, the $C B S$-KO rabbits were fed with vitamin $\mathrm{B}$ and betaine complex supplements to devise possible therapeutic approaches.

\section{Material and methods}

\section{Construction of the CRISPR/Cas9 system}

The CRISPR/Cas9 single guide RNAs (sgRNAs) for rabbit $C B S$ were designed using http://crispr.mit.edu based on its sequence (Genbank: NW_003160195.1; http://www.ncbi. nlm.nih.gov/). Three CBS-targeting sgRNAs were screened (sgRNA1 sited E8: g.6631-6650, sgRNA2 sited E8: g.66376656 and sgRNA3 sited E8: 6641-6660) (Fig. 1). The detailed protocol has been described previously [41, 42]. Single-stranded oligodeoxynucleotide donor templates (ssODN) with silent mutations were designed and synthesized by Shanghai Sangon Biotechnology Co. Ltd. as follows: GGCTCCATCCTGGCG GAGCCGGAGGAGC TGAA CCAGACGGAGGTGACGGCCTAtGAaGTaGAa GGtATC tcCTACGACTTCATC CCCACCGTGCTCGA C CGGACGGTGTGT GGGCCCCAG.

\section{Zygote injection with Cas9/sgRNA and embryo transfer}

New Zealand White rabbits (6-8 months old) were housed at the Animal Genetic Engineering Laboratory of Yangzhou University under a $12 \mathrm{~h}$ diurnal/ nocturnal cycle, and fed twice a day with free access to water. All protocols were approved by the Care 
A

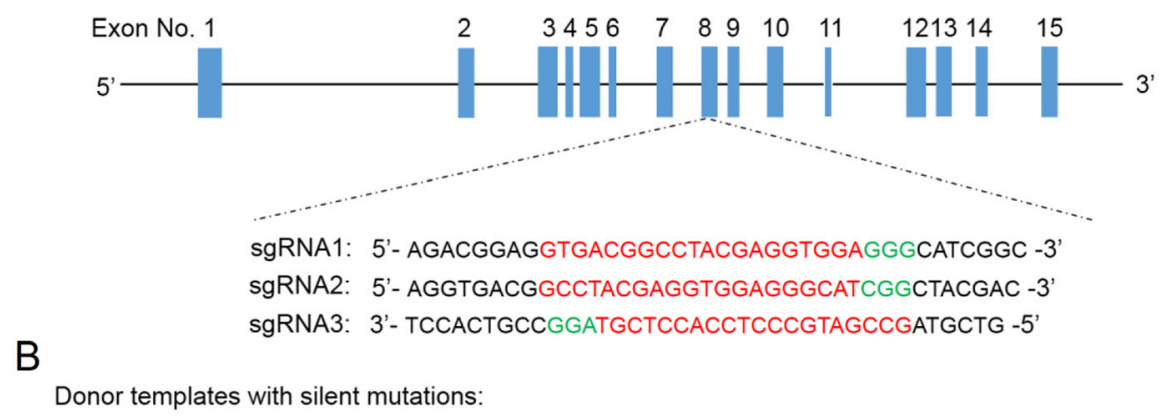

GGCTCCATCCTGGCGGAGCCGGAGGAGCTGAACCAGACGGAGGTGACGGCCTATGAAGTAGA

AGGTATCTCCTACGACTTCATCCCCACCGTGCTCGACCGGACGGTGTGTGGGCCCCAG $\overline{\mathrm{G} 307 \mathrm{~S}}$

Fig. 1 Homology-dependent repair (HDR) knock-in of CBS gene in rabbits using CRISPR/Cas9 gene editing. a CRISPR/Cas9-targeting sites on the rabbit CBS gene. The red letters in the sgRNA sequences are specific for exon 8, and the green letters indicate the protospacer adjacent motif (PAM). b Donor template sequences with silent mutations (green letters) and G307S (red letters)

and Use of Laboratory Animals (Ministry of Science and Technology of the People's Republic of China) and the Animal Care and Use Committee of Yangzhou University, Yangzhou, China (license number: SYXK(Su)2017-0044).

Female rabbits were superovulated by intramuscular injection of follicle-stimulating hormone (Sansheng Pharmaceutical Co., Ltd., NingBo, China) twice daily. The dosage was $15 \mathrm{IU}$ for the first two injections, $10 \mathrm{IU}$ for the next two and $5 \mathrm{IU}$ for the last two injections. After the final injection, both the superovulated and recipient females were injected with $100 \mathrm{IU}$ human chorionic gonadotropin (HCG), and the former were mated with male rabbits. Approximately $18-20 \mathrm{~h}$ post-coitus, the female rabbits were anesthetized with pentobarbital $(2 \%, 20 \mathrm{mg} / \mathrm{kg}$, i.p.), and the pronuclear zygotes were extracted and transferred into M2 medium (SigmaAldrich,

St. Luis, MO, USA) supplemented with $10 \%$ fetal bovine serum (Hyclone, Logan, UT, USA). Following cytoplasmic injection of $40 \mathrm{ng} / \mu \mathrm{L}$ Cas 9 mRNA, $10 \mathrm{ng} /$ $\mu \mathrm{L}$ of an sgRNA and $25 \mathrm{ng} / \mu \mathrm{L}$ ssODN, the embryos were transferred to complete (with 10\% FBS) M16 medium (Sigma-Aldrich, St. Luis, MO, USA) and incubated at $38{ }^{\circ} \mathrm{C}$ under $5 \% \mathrm{CO}_{2}$ for $30-60 \mathrm{~min}$. Approximately $15-20$ embryos were transferred to one recipient female.

\section{Genotypic analysis}

Genomic DNA was extracted from ear biopsies via phenol-chloroform extraction, and the $C B S$ sequences were amplified using specific primers (Table 1). The amplified products were extracted and purified from the gel using a PCR Purification kit (Transgene Biotechnology Co., Ltd., Beijing, China). The sequences were then cloned into pGEM-T vector (Promega, Madison, WI, USA), and sequenced by Shanghai Sangon Biotechnology Co. Ltd. Sequences were analyzed by the Lasergene DNA analysis package (DNAStar Inc., Madison, WI, USA).

To determine any off-target effects, the CRISPR design tool (http://tools.genomeengineering.org) was used to predict potential sites homologous to the 23-bp sgRNA + PAM sequence across the rabbit genome. These sites were then amplified in the genomic DNA of founder CBS-KO rabbits and sequenced. The off-target sites and primer pairs are listed in Supplementary Tables 1 and 2 (Table S1, Table S2).

\section{Drug test}

Two weeks-old CBS-KO and WT rabbits were infused daily with $2.5 \mathrm{mg} / \mathrm{kg}$ vitamin $\mathrm{B} 6,25 \mu \mathrm{g} / \mathrm{kg}$ vitamin B12, $45 \mu \mathrm{g} / \mathrm{kg}$ folate and $25 \mathrm{mg} / \mathrm{kg}$ betaine via the gastric route. The animals were acclimatized to gentle restraint and handling, and infused daily from 10 am to 12 noon for 4 weeks by the same technician.

Table 1 Primers for detection of CBS -KO rabbits

\begin{tabular}{lll}
\hline Name & $\mathbf{5}^{\prime}-\mathbf{3}^{\prime}$ & TM \\
\hline CBS-1 & GCTGGCTTCAGTCAGGTTC & $55.1^{\circ} \mathrm{C}$ \\
CBS-2 & CTCCTTGTCGGTGCTCTTG & $56.1^{\circ} \mathrm{C}$ \\
\hline
\end{tabular}




\section{Biochemical analysis}

Four milliliters peripheral blood was collected into EDTA-coated tubes from each animal after withholding food for 10-12 h. Plasma was separated by centrifuging the blood at $3000 \mathrm{rpm}$ at $4{ }^{\circ} \mathrm{C}$. The levels of triglycerides (TG), total cholesterol (TC), and high-density (HDL-C) and low-density lipoprotein cholesterol (LDL-C) were measured using specific kits (A110-1, A111-1, A112-1 and A113-1; Nanjing Jiancheng Bioengineering Institute, Nanjing, China). In addition, Hcy levels were measured using an ELISA kit (Laier Biotechnology Co., Ltd., Hefei, China).

\section{Western blotting}

The expression levels of the apolipoproteins (Apo) B, E and A-I in the plasma were analyzed by Western blotting as per standard protocols. The protein bands were probed with the goat anti-ApoE and anti-ApoB (both from Rockland, Limerick PA), and sheep antiApoA-I (AbD Serotec, Oxford, UK) antibodies. The secondary antibodies were HRP-conjugated donkey anti-goat IgG (Jackson Immuno Research Laboratories, West Grove, PA, US) and donkey anti-sheep IgG (Chemicon, Temecula, CA, US).

\section{Histomorphological assessment}

The CBS-KO and WT littermates were maintained under similar conditions, and fed according to their growth stage. The body weight of the rabbits was monitored from birth till 6 weeks of age. After euthanizing the animals with sodium pentobarbital overdose, the liver lobes were harvested and fixed in $4 \%$ paraformaldehyde. For histological analysis, the fixed tissues were embedded in paraffin and cut into sections that were stained using hematoxylin and eosin (HE) using standard protocols. The stained sections were viewed under a Leica light microscope.

\section{Statistical analysis}

Data were expressed as mean $\pm \mathrm{SD}$ and compared by Student's t test. GraphPad Prism was used for all statistical analyses, and $P<0.05$ was considered statistically significant.

\section{Results}

\section{Generation of CBS-KO rabbits and genotypic analysis}

As shown in Table 2, we injected 217 zygotes with the CRISPR/Cas9 and sgRA constructs, and transferred 181 into 9 surrogate females. Eighteen pups harbored a mutant CBS (Table 2), of which only 6 survived and were

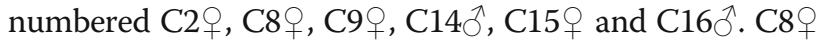
was homozygous for G307S, C2우 harbored a frameshift mutation in $C B S$ due to deletion or substitution, and $C 9$, $\mathrm{C} 14{ }^{\wedge}$ and one allele of $\mathrm{C} 16{ }^{\lambda}$ harbored deletions/
Table 2 Generation of CBS-KO rabbits using CRISPR/Cas9

\begin{tabular}{ll}
\hline Number of injected zygotes & 217 \\
Number of transferred zygotes & 181 \\
Number of recipients & 9 \\
Number of pregnancies & 5 \\
Total births & 18 \\
Number of mutants & 18 \\
Number of G307S mutants & 1 \\
Pregnancy rate & $55.56 \%$ \\
Mutation rate & $100 \%$ \\
G307S rate & $5.56 \%$ \\
\hline
\end{tabular}

substitutions in the $C B S$ gene without a frameshift in the amino acid sequence. The second $C B S$ allele of $C 16{ }^{-1}$ had deletions/insertions resulting in a frameshift mutation. One allele of $\mathrm{C} 159$ harbored the G307S and the other showed deletions/insertions resulting in frameshift mutation (Fig. 2). In addition, 15 potential off-target sites (OTs, five for each sgRNA) were also amplified and sequenced, and no overlapping peaks were detected near the OTs.

\section{Mutations in CBS induced hyperlipidemia which was reversed by betaine}

Since HHcy is closely associated with dyslipidemia, we next analyzed the levels of blood lipids in the WT and mutant rabbits. As shown in Table 3, the $\mathrm{C} 29$ and $\mathrm{C} 8$ ? rabbits fed with normal chow had significantly higher serum levels of Hcy, TG, TC and LDL-C compared to the age-matched WT rabbits. The Hcy levels in $\mathrm{C} 2$ 우 $(48.80 \mu \mathrm{mol} / \mathrm{L})$ and $\mathrm{C} 8$ ㅇ $(52.66 \mu \mathrm{mol} / \mathrm{L})$ were almost twice as high as that in WT controls, whereas the TG levels $(3614.93 \mathrm{mg} / \mathrm{dL}$ and $3878.47 \mathrm{mg} / \mathrm{dL}$ respectively) showed a 50-54 fold increase. The TC levels in $\mathrm{C} 2$ 오 $(738.98 \mathrm{mg} / \mathrm{dL})$ and $\mathrm{C} 89(342.62 \mathrm{mg} / \mathrm{dL})$ were respectively 6 - and 3 -fold higher, and that of LDL-C levels in both $(67.24 \mathrm{mg} / \mathrm{dL}$ and $76.80 \mathrm{mg} / \mathrm{dL}$ respectively) were 2-fold higher compared to the WT. In contrast, the HDL-C level in $\mathrm{C} 8$ + $(16.64 \mathrm{mg} / \mathrm{dL})$ was significantly lower compared to the WT. Consistent with the high levels of circulating lipids in $\mathrm{C} 8$ \% , the color of its plasma was milky white (Fig. 4b).

To evaluate a potential hypolipidemic effect of betaine, we infused both WT and mutant animals with vitamin B and betaine complex for 4 weeks. Betaine supplementation reduced plasma Hcy, TG, TC and LDL-C by $30.50 \%$ $(19.41 \pm 1.77 \mu \mathrm{mol} / \mathrm{L} \quad$ vs $\quad 27.93 \pm 2.47 \mu \mathrm{mol} / \mathrm{L}), \quad 36.88 \%$ $(45.00 \pm 7.16 \mathrm{mg} / \mathrm{dL} \quad$ vs $\quad 71.31 \pm 6.70 \mathrm{mg} / \mathrm{dL}), \quad 27.31 \%$ $(89.33 \pm 4.94 \mathrm{mg} / \mathrm{dL}$ vs $122.89 \pm 11.83 \mathrm{mg} / \mathrm{dL})$ and $39.33 \%$ $(17.14 \pm 0.56 \mathrm{mg} / \mathrm{dL}$ vs $28.25 \pm 3.54 \mathrm{mg} / \mathrm{dL})$ respectively in the WT rabbits compared to non-supplemented littermates. In contrast, no significant changes were seen in plasma HDL-C levels (Fig. 3a). The similarly fed C9우, 


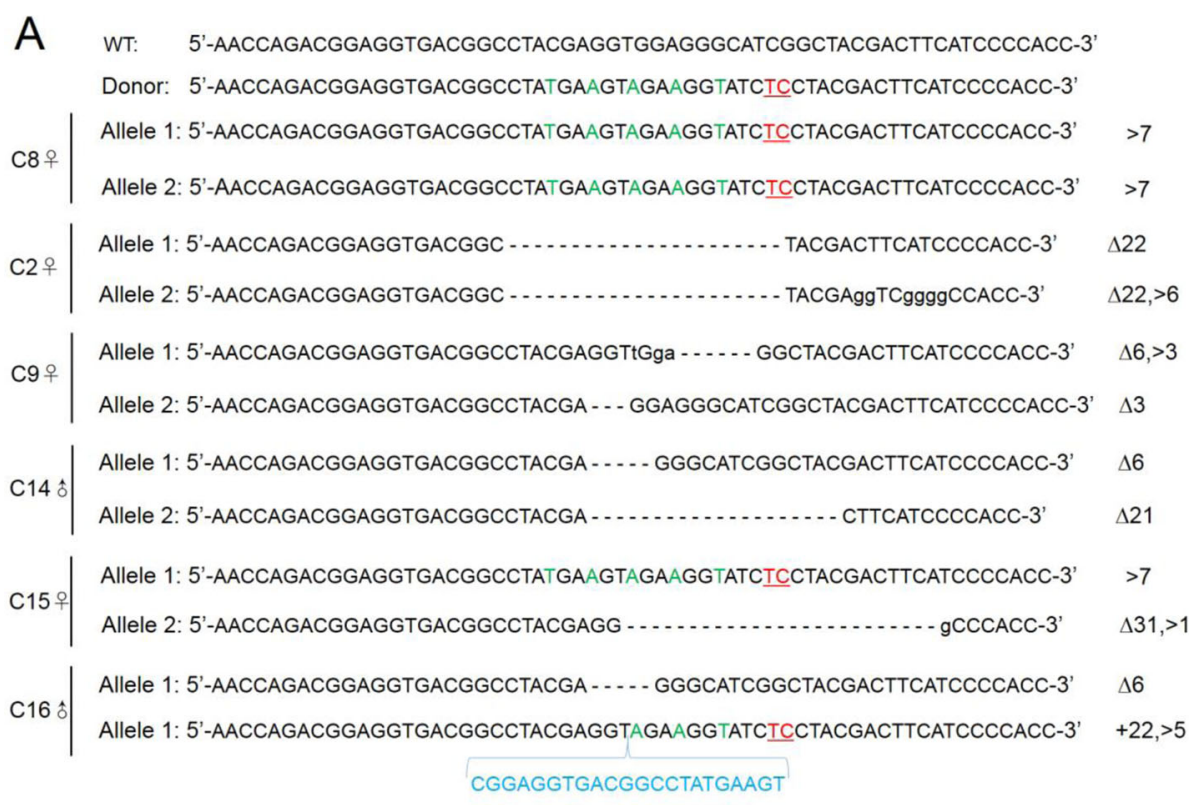

B 307

WT: PEELNQTEVTAYEVEGIǴYDFIPTVLDRTVVDRWFKSTDKEAFAFARMLIAQEGLLCGGS

$C 8 \propto \mid \begin{array}{ll}\text { Allele1:PEELNQTEVTAYEVEGIŠYDFIPTVLDRTVVDRWFKSTDKEAFAFARMLIAQEGLLCGGS } & >1 \\ \text { Allele2:PEELNQTEVTAYEVEGIŚYDFIPTVLDRTVVDRWFKSTDKEAFAFARMLIAQEGLLCGGS } & >1\end{array}$

C2 $ๆ \mid \begin{aligned} & \text { Allele1:PEELNQTEVTATTSSPPĆSTGRWWTGGSRAPTRRPSPSPAC. } \\ & \text { Allele2:PEELNQTEVTATRSGPPĆSTGRWWTGGSRAPTRRPSPSPAC. }\end{aligned}$

C9ㅇำ Allele1:PEELNQTEVTAYEV - G - GYDFIPTVLDRTVVDRWFKSTDKEAFAFARMLIAQEGLLCGGS $\Delta 2$

Allele2:PEELNQTEVTAYE - EGIGÝDFIPTVLDRTVVRWFKSTDKEAFAFARMLIAQEGLLCGGS $\Delta 1$

| Allele1:PEELNQTEVTAY - - EGIGY'́rIPTVLDRTVVDRWFKSTDKEAFAFARMLIAQEGLLCGGS $\quad \Delta 2$

C14

Allele2:PEELNQTEVTA . . . . . . . YDFIPTV̌LDRTVVDRWFKSTDKEAFAFARMLIAQEGLLCGGS

C15요 Allele1:PEELNQTEVTAYEVEGISYDFIPTVLDRTVVDRWFKSTDKEAFAFARMLIAQEGLLCGGS >1

Allele2:PEELNQTEVTAYEGPPCŚTGRWWTGGSRAPTRRPSPSPAC.

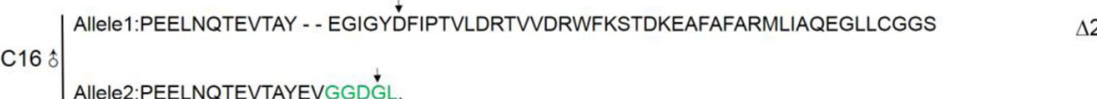

$>1$

Fig. 2 Generation of mutant CBS alleles by injecting rabbit zygotes with CRISPR/Cas9. a Types of mutations in the CBS alleles in founder rabbits. The WT and donor ssODN template sequences are shown at the top. Silent mutations, point mutations, substitutions ( $>$ ) and insertions (+) are labeled with green letters, red underlined letters, black lowercase letters and blue letters respectively. The deletions $(\Delta)$ are indicated on the right of the respective allele. $\mathbf{b}$ Theoretical amino acid sequences of the founder rabbits. The amino acid at 307 is shown with a black arrow. Deletions ( $\triangle$ ) and substitutions $(>)$ are shown to the right of each allele

$\mathrm{C} 14 \hat{\jmath}, \mathrm{C} 159$ and $\mathrm{C} 16 \hat{\jmath}$ mutants showed a significant decrease in plasma levels of TG, TC, LDL-C and Hcy compared to that in $\mathrm{C} 29$ and $\mathrm{C} 8$ \% . In addition, the TG levels in C14 $\overbrace{}^{\lambda}(76.14 \mathrm{mg} / \mathrm{dL})$ and $\mathrm{C} 15$ ㅇ $(35.95$ $\mathrm{mg} / \mathrm{dL})$, and the LDL-C levels in $\mathrm{C} 16{ }^{-}(24.64 \mathrm{mg} / \mathrm{dL})$ dropped to near normal range after betaine supplementation (Tables 3 and 4). Consistent with these observations, the $\mathrm{C} 2 q$ and $\mathrm{C} 8 q$ rabbits showed a significant increase in $\mathrm{ApoE}$ and $\mathrm{ApoB}$ compared to the WT, whereas betaine supplementation in $\mathrm{C} 9$ 웅 
Table 3 Plasma Hcy and lipid profile of the rabbits without vitamin B and betaine supplementation

\begin{tabular}{llllll}
\hline Cholesterol & Hcy $(\boldsymbol{\mu m o l} / \mathbf{L})$ & TG $(\mathbf{m g} / \mathbf{d L})$ & TC $(\mathbf{m g} / \mathbf{d L})$ & HDL-C $(\mathbf{m g} / \mathbf{d L})$ & $\mathbf{L D L}-\mathbf{C}(\mathbf{m g} / \mathbf{d L})^{\mathbf{a}}$ \\
\hline WT $(n=6)$ & $27.93 \pm 3.49$ & $71.31 \pm 13.39$ & $122.89 \pm 23.66$ & $38.53 \pm 1.73$ & $28.25 \pm 7.09$ \\
C2ㅇ & 48.80 & 3614.93 & 738.98 & 91.33 & 67.24 \\
C8ㅇ & 52.66 & 3878.47 & 342.62 & 16.64 & 76.80
\end{tabular}

Values are expressed as means \pm SD. ${ }^{a}$ The data does not contain vLDL-C

$\mathrm{C} 14 \hat{\circ}, \mathrm{C} 15$ 우 and $\mathrm{C} 16 \hat{\circ}$ reversed these trends (Fig. 3c).

\section{The morphology of CBS-KO rabbits}

The body weights of the WT and CBS-KO rabbits were similar on postnatal days 1 and 7. At 3 weeks however, the $C B S-K O$ rabbits weighed significantly less compared to the WT animals (Table 5), and some failed to gain weight even after 6 weeks. In addition, CBS-KO rabbits were overall smaller, and had sparser fur and pale mucous membranes (Fig. 4c-e), all of which are indicative of growth retardation. The eye-lens were also dislocated in the CBS-KO rabbits (Fig. 4a). Finally, most of the mutant animals did not survive beyond $5 \sim 7$ weeks after birth (Table 6).

\section{Histological analysis}

The 6-week-old WT and CBS-KO rabbits receiving normal and vitamin $\mathrm{B} /$ betaine complex-supplemented feed were sacrificed for histological examination. The livers of $C B S-\mathrm{KO}$ rabbits were brick red compared to the reddish-brown color of the WT livers (Fig. 5a).

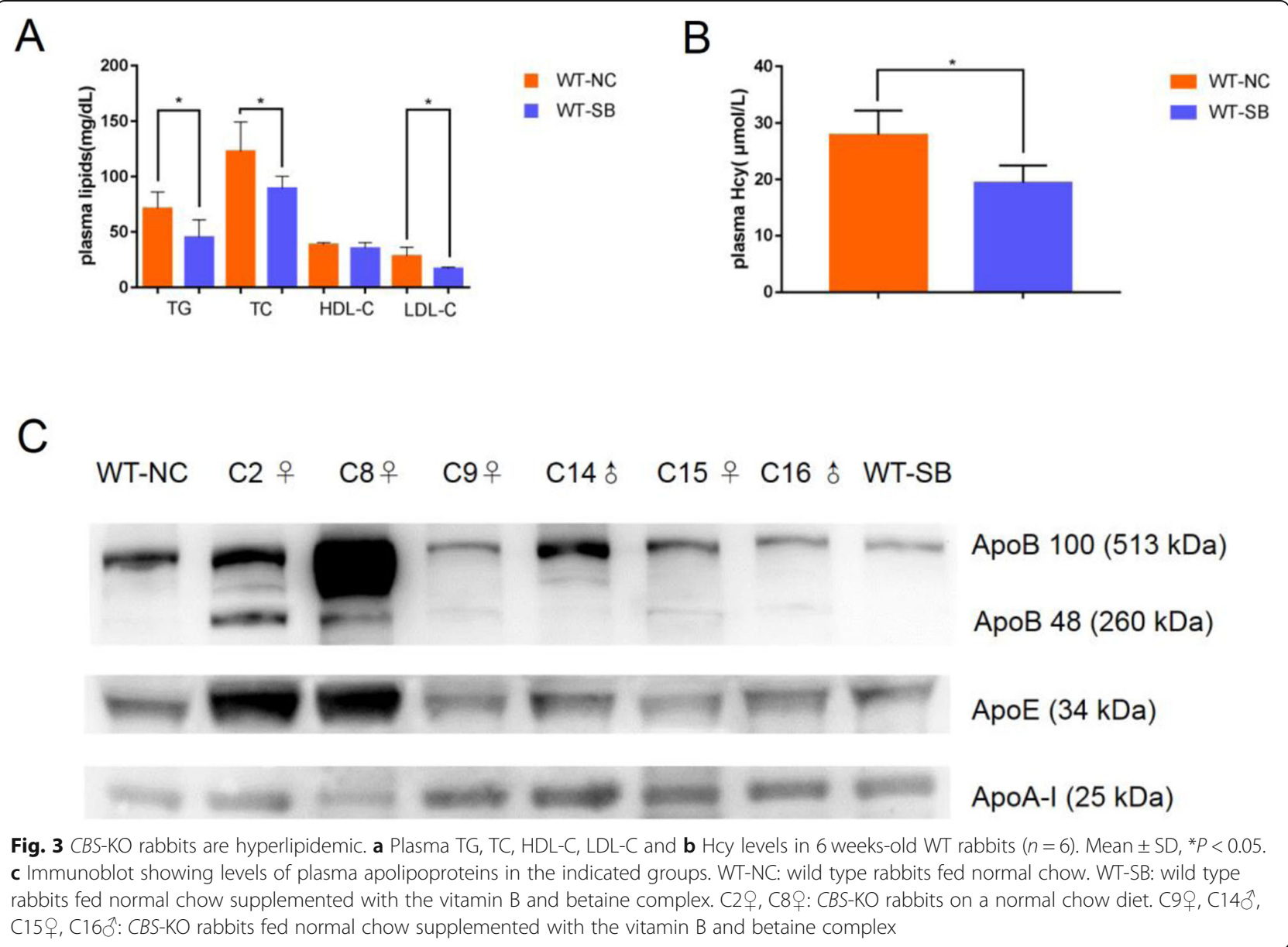


Table 4 Plasma Hcy and lipid profile of the rabbits with vitamin B and betaine supplementation

\begin{tabular}{llllll}
\hline Cholesterol & Hcy $(\boldsymbol{\mu m o l} / \mathbf{L})$ & TG $(\mathbf{m g} / \mathbf{d L})$ & TC $(\mathbf{m g} / \mathbf{d L})$ & HDL-C $(\mathbf{m g} / \mathbf{d L})$ & $\mathbf{L D L}-\mathbf{C}(\mathbf{m g} / \mathbf{d L})^{\mathbf{a}}$ \\
\hline WT(n=6) & $19.41 \pm 2.50$ & $45.00 \pm 14.35$ & $89.33 \pm 9.88$ & $35.24 \pm 4.57$ & $17.14 \pm 1.11$ \\
C99 & 26.40 & 103.27 & 36.55 & 3.48 & 15.35 \\
C140 & 25.06 & 76.14 & 293.89 & 19.48 & 88.02 \\
C159 & 25.25 & 35.95 & 160.09 & 27.48 & 61.66 \\
C160 & 30.8 & 22.03 & 61.87 & 23.22 & 24.64 \\
\hline
\end{tabular}

Values are expressed as means \pm SD. ${ }^{\mathrm{a}}$ The data does not contain vLDL-C

Histologically, no cytoplasmic lipid droplets were observed in the liver of WT rabbits even in the absence of vitamin $B$ and betaine complex supplementation. However, the $C B S-\mathrm{KO}$ rabbits had significant accumulation of macro cytoplasmic lipid droplets in their liver, which decreased following vitamin B and betaine complex supplementation (Fig. 5b). However, Sudan IV staining did not reveal any atheromatous plaques in the aorta and other vascular segments of the CBS-KO rabbits (Fig. 5c).

\section{Discussion}

Homocysteinemia is observed in $5-30 \%$ of the human population, and leads to vascular complications in onefourth of the patients [43-45]. We obtained 6 CBS-KO rabbits including one harboring the G307S point mutation, using the CRISPR/Cas9 gene editing technology. All mutants $(\mathrm{C} 2 \%$ and $\mathrm{C} 8$ \% $)$ lacked the glycine 307 residue in CBS protein, and displayed high serum levels of Hcy and lipids. Supplementing the normal chow with vitamin $B$ and betaine alleviated the pathological symptoms of HHcy. Although there was some research reported on the G307S point mutation, it was mostly based on the heterologous gene expression system such as E. coli, S. cerevisiae, and Chinese hamster ovary cells. But it was unfortunate that the heterologous $\mathrm{CBS}^{\mathrm{G} 307 \mathrm{~S}}$ protein lacked enzyme activity [46-48]. At present, there was no CBS-deficient animal model of G307S point mutation or deletion of glycine at position 307 . The CBS-
KO rabbit showed HHcy, dyslipidemia, a short life span, hepatic steatosis and other lesions. This indicates that HHcy and hyperlipidemia are likely the result of 307G deletion as well as $\mathrm{G} \rightarrow \mathrm{S}$ mutation. Compared with $C B S$-deficient mice in the previous research $[49,50]$, the results of this study may be more precise and accurate in explaining the molecular mechanism of hereditary hyperhomocysteinemia etiology. Therefore, the CBS-KO rabbit model is suitable for studying the pathogenesis and therapeutic strategies of HHcy. Studies show that HHcy is an independent risk factor for CVDs [3], which correlates positively with atherosclerotic symptoms induced by the high levels of TG, TC and LDL-C, and normal plasma HDL-C level [51, 52]. In addition, HHcy increases the risk of hypertriglyceridemia [53, 54]. Consistent with this, the serum triglyceride levels were high in the $C B S-\mathrm{KO}$ founder rabbits, and decreased significantly following betaine and vitamin B6 supplementation.

Dyslipidemia is a major factor underlying CVDs, and normalizing blood lipid levels can lower the morbidity and mortality in patients with heart disease [55]. The quaternary amine betaine and B vitamins are efficient methyl donors that produce a large amount of free carnitine in the liver, which promotes long-chain ester acylCoA entry into the mitochondria and accelerates fatty acid oxidation, eventually reducing the blood lipid levels [56]. Betaine may inhibit hepatic biosynthesis of LDL-C

Table 5 Growth of CBS-KO and WT rabbits from 1 to 42 days old

\begin{tabular}{|c|c|c|c|c|c|c|c|c|}
\hline \multirow{2}{*}{$\begin{array}{l}\text { Postpartum } \\
\text { age, days }\end{array}$} & \multicolumn{8}{|c|}{ Body weight, g } \\
\hline & $\overline{\mathbf{C 2}}+$ & $\mathbf{C 8}+$ & $\mathrm{C9}+$ & C24 & $\mathrm{C} 25$ 우 & $\mathrm{C} 26{ }^{\wedge}$ & CBS-KO $(n=6)$ & WT $(n=6)$ \\
\hline 1 & 58 & 67 & 60 & 52 & 61 & 59 & $59.50 \pm 4.43$ & $55.17 \pm 3.28$ \\
\hline 7 & 73 & 84 & 83 & 77 & 88 & 68 & $78.83 \pm 6.87$ & $88.33 \pm 6.97$ \\
\hline 14 & 123 & 112 & 124 & 102 & 113 & 118 & $115.33 \pm 7.48^{*}$ & $133.17 \pm 15.24$ \\
\hline 21 & 161 & 176 & 177 & 129 & 169 & 165 & $162.83 \pm 16.15^{* *}$ & $208.6 \pm 22.48$ \\
\hline 28 & 214 & 216 & 234 & 205 & 240 & 236 & $224.17 \pm 13.06^{* *}$ & $278.3 \pm 30.60$ \\
\hline 35 & 310 & 306 & 300 & 302 & 350 & 353 & $320.17 \pm 22.39^{* *}$ & $359.5 \pm 28.58$ \\
\hline 42 & 382 & 397 & 425 & 413 & 438 & 453 & $418 \pm 23.93^{* * * *}$ & $590.6 \pm 40.86$ \\
\hline
\end{tabular}

Values are expressed as means \pm SD. ${ }^{*} P<0.05,{ }^{* *} P<0.01$ and ${ }^{* * *} P<0.0001$ 

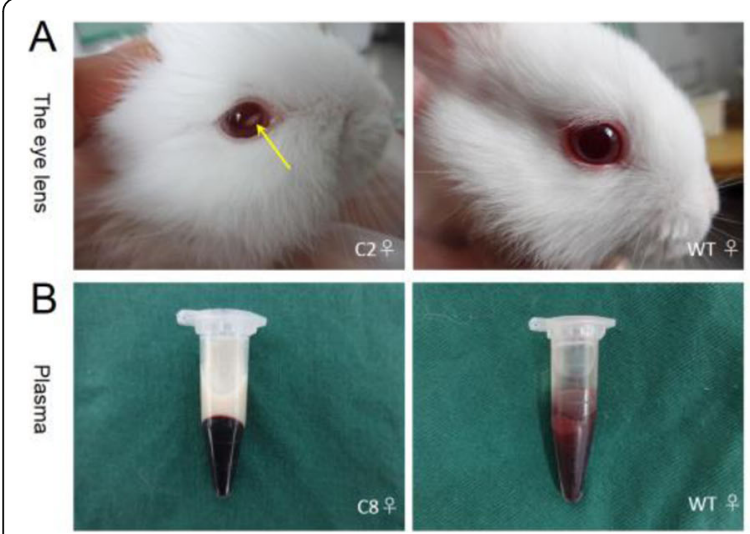

C

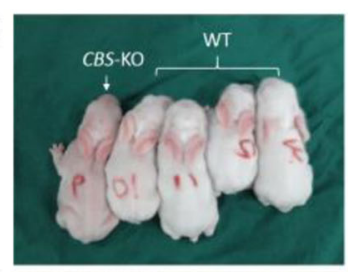

D

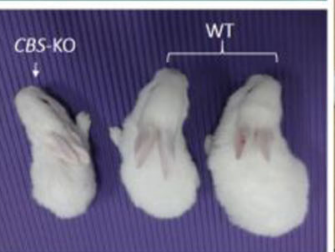

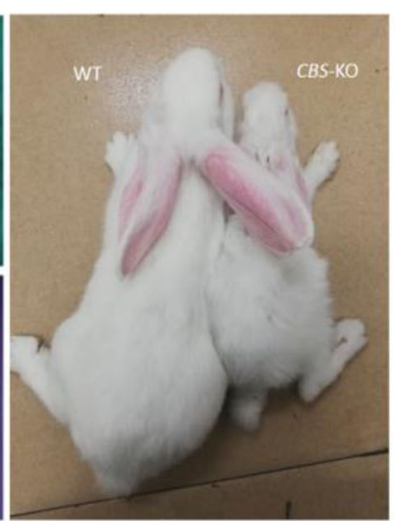

$\mathrm{E}$

Fig. 4 The CBS-KO rabbits showed significant growth retardation. a Representative images of the eyes indicating lens dislocation (yellow arrow) in the C29 rabbit. b Representative pictures of the milky white plasma of C8ㅇ (G307S) and transparent plasma of WT rabbits. Representative images of the CBS-KO and WT rabbits at (c) 1 week, (d) 3 weeks and (e) 9 weeks

or accelerate the conversion of LDL to HDL [57]. In addition, oral betaine and $B$ vitamins can reduce serum Hcy levels in healthy individuals by increasing their metabolism, and have therapeutic effects on patients with hyperlipidemia, fatty liver and atherosclerosis [58-60]. Consistent with this, vitamin B and betaine complex supplementation significantly improved the blood lipid profile of both WT and CBS-KO rabbits. Interestingly, the normal rabbits also showed Hcy levels higher than $15 \mu \mathrm{mol} / \mathrm{L}$, which may explain the rapid induction of hyperlipidemia via high-fat diet consumption. Thus, the species and nutrient intake should be taken into account when assessing the effect of Hcy metabolism on dyslipidemia.

The fatty liver parenchyma of CBS-KO rabbits was filled with microvesicular cytoplasmic lipid droplets due to the influence of $\mathrm{Hcy}$ on the expression level of Smethyltransferase (BHMT). CBS-KO rabbits showed signs of growth retardation and short survival. Betaine and vitamin B supplementation reduced the fatty liver symptoms and prolonged survival of the mutants. Maclean [61] et al. reported that transgenic expression of $C B S$ alleviated liver steatosis and prolonged survival in a mouse model. We showed for the first time that the addition of betaine and $\mathrm{B}$ vitamins partly compensated for the lack of $C B S$. This is significant since the previously reported hypolipidemic effects of betaine were seen animal models with high-fat diet-induced hyperlipidemia.

Table 6 The survival duration of CBS-KO rabbits

\begin{tabular}{lllllll}
\hline & $\mathbf{C 2}+$ & $\mathbf{C 8}+$ & $\mathbf{C 9}+$ & $\mathbf{C 2 4}^{\wedge}$ & $\mathbf{C 2 5}+$ & $\mathbf{C 2 6}$ \\
\hline days & 46 & 43 & 50 & 66 & 109 & 52 \\
\hline
\end{tabular}

HHcy is an important factor in hyperlipidemia and cardiovascular disease. In this study, animal models of HHcy showed hyperlipidemia and cardiovascular disease symptoms. Although the lipoprotein metabolism and cardiovascular pathophysiology of rabbits are similar to that of humans [62], the inherently higher Hcy levels in rabbits may have resulted in premature death in the absence of $C B S$.

Compared to the untreated animals, the CBS-KO rabbits with betaine and vitamin $B$ supplementation had lower ApoB100, ApoB48 and ApoE levels in their plasma. ApoB is the major component of very low density lipoprotein (vLDL) and LDL particles. Namekata et al. [49] detected elevated ApoB in $\mathrm{CBS}^{-1-}$ mouse serum, and Salahi et al. [63] found that betaine supplementation decreased LDL concentration and increased that of HDL in a rat model. ApoB is abundant in human and rabbit but not murine plasma [64]. In addition, the chemical composition and cholesterol ester transfer subunits of human and rabbit ApoB-related lipoproteins are similar, which correlates with the role of rabbit ApoB in atherosclerosis [65].

In conclusion, CBS-KO rabbits have impaired growth and metabolism, although it remains to elucidated whether these changes are the direct effect of the mutation, or an indirect effect of hyperlipidemia or high homocysteine.

\section{Study strengths and limitations}

We have generated CBS-KO rabbits with G307S mutation for the first time using the CRISPR/Cas9 system, and the model exhibits hyperhomocysteinemia and 


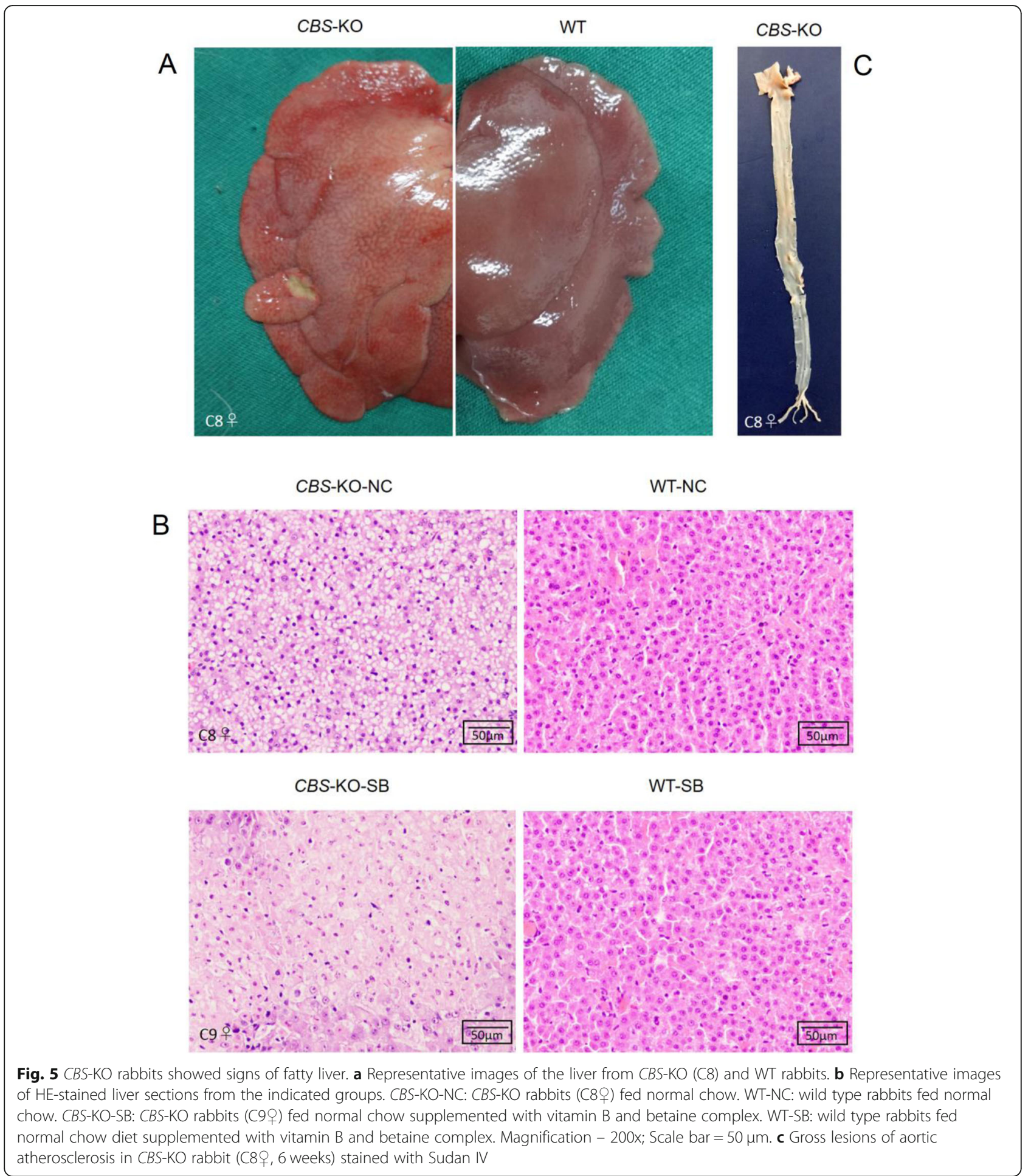

dyslipidemia on a normal chow diet. It is also the first report on the effect of betaine on the serum lipids of CBS-KO rabbits.

However, the short lifespan of these rabbits obviated

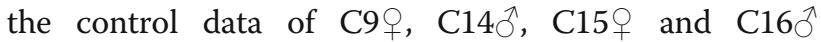

without betaine and vitamin B complex supplementation. Nevertheless, Hcy, TG, TC, LDL-C and other indices of most $C B S-\mathrm{KO}$ rabbits were higher compared to that of the normal controls even after betaine and vitamin B complex supplementation. 


\section{Conclusion}

In conclusion, the $C B S$ mutated rabbit model is a promising tool for studying human dyslipidemia, despite their high mortality. The lesions of G307S homozygous CBS-KO rabbit should be useful to clearly understand the pathophysiology of the CBS gene deficiency and the G307S mutation rabbit provides a suitable animal model for drug development.

\section{Supplementary information}

Supplementary information accompanies this paper at https://doi.org/10. 1186/s12944-020-01394-5.

Additional file 1: Table S1. The sequences of potential off-target sites. Additional file 2: Table S2. The sequences of potential off-target loci PCR primers.

\section{Abbreviations}

HHcy: Hyper-homocysteinemia; CBS: Cystathionine $\beta$-synthase; TG: Triglycerides; TC: Total cholesterol; LDL-C: Low-density lipoprotein cholesterol; HDL-C: High-density lipoprotein cholesterol; VLDL-C: Very Lowdensity lipoprotein cholesterol; CVDs: Cardiovascular diseases; ApoB: Apolipoprotein B; APOE: Apolipoprotein E

\section{Acknowledgments}

This study was supported by the National Key Research and Development Program of China, China (No. 2016YFE0126000), the Priority Academic Program Development of Jiangsu Higher Education Institutions, China (PAPD) and the Yangzhou University-enterprise Cooperation Project, China (YZ2017283)

\section{Authors' contributions}

Yong Cheng and Jingyan Liang designed the experiments and provided the resources. Ting Zhang, Rui Lu, and Yong Cheng performed the experiments, analyzed the data, and wrote the manuscript. Ting Zhang, Yibing Chen, Yuguo Yuan, Shaozheng Song, Kunning Yan, Yiwen Zha and Wenwen Zhuang performed the experiments and collected data. All authors have read and approved the final paper.

\section{Competing interests}

The authors declare no conflicts of interest.

\section{Author details}

${ }^{1}$ College of Veterinary Medicine, Yangzhou University, Yangzhou 225009, Jiangsu, China. ${ }^{2}$ Jiangsu Co-innovation Center for Prevention and Control of Important Animal Infectious Diseases and Zoonoses, Yangzhou 225009, Jiangsu, China. ${ }^{3}$ School of Pharmacy, Jiangsu Food \& Pharmaceutical Science College, Huaian 223003, Jiangsu, China. ${ }^{4}$ School of Nursing, Taihu University of Wuxi, Wuxi 214000, Jiangsu, China. ${ }^{5}$ Institute of Translational Medicine, Medical College, Yangzhou University, Yangzhou 225001, Jiangsu, China.

\section{Received: 30 July 2020 Accepted: 28 September 2020}

Published online: 14 October 2020

\section{References}

1. Hansrani M, Gillespie Jl, Stansby G. Homocysteine in myointimal hyperplasia. Eur J Vasc Endovasc Surg. 2002;23(1):3-10

2. Mudd SH, et al. Homocystinuria: an enzymatic defect. Science. 1964; 143(3613):1443-5.

3. Clarke R, et al. Hyperhomocysteinemia: an independent risk factor for vascular disease. N Engl J Med. 1991;324(17):1149-55.

4. Audelin MC, Genest J Jr. Homocysteine and cardiovascular disease in diabetes mellitus. Atherosclerosis. 2001;159(2):497-511.

5. Werstuck $\mathrm{GH}$, et al. Homocysteine-induced endoplasmic reticulum stress causes dysregulation of the cholesterol and triglyceride biosynthetic pathways. J Clin Invest. 2001;107(10):1263-73.

6. McCully KS. Homocysteine and vascular disease. Nat Med. 1996;2(4):386-9.
7. den Heijer M, et al. Hyperhomocysteinemia and venous thrombsis: a metaanalysis. Thromb Haemost. 1998;80(6):874-7.

8. Boers $\mathrm{GH}$, et al. Heterozygosity for homocystinuria in premature peripheral and cerebral occlusive arterial disease. N Engl J Med. 1985;313(12):709-15.

9. McCully KS. Homocysteine and the pathogenesis of atherosclerosis. Expert Rev Clin Pharmacol. 2015;8(2):211-9.

10. Zhang $\mathrm{S}$, et al. Association between serum homocysteine and arterial stiffness in elderly: a community-based study. J Geriatr Cardiol. 2014;11(1): 32-8

11. Yang $X$, et al. Homocysteine and Carotid Plaque Stability: A Cross-Sectional Study in Chinese Adults. PLoS One. 2014:9(4).

12. Williams KT, Schalinske KL. Homocysteine metabolism and its relation to health and disease. Biofactors. 2010;36(1):19-24.

13. Kang SS, Wong PW, Malinow MR. Hyperhomocyst(e)inemia as a risk factor for occlusive vascular disease. Annu Rev Nutr. 1992;12:279-98.

14. Zhou J, Austin RC. Contributions of hyperhomocysteinemia to atherosclerosis: causal relationship and potential mechanisms. Biofactors. 2009;35(2):120-9.

15. Gaiday AN, et al. Effect of homocysteine on pregnancy: a systematic review Chem Biol Interact. 2018;293:70-6.

16. Kraus JP, Kozich V. Pyridoxine responsive and unresponsive homocystinuria. J Nutr Sci Vitaminol (Tokyo). 1992. Spec No:589-92.

17. Kruger WD, Cox DR. A yeast assay for functional detection of mutations in the human cystathionine beta-synthase gene. Hum Mol Genet. 1995;4(7): 1155-61.

18. Kraus JP. Komrower lecture. Molecular basis of phenotype expression in homocystinuria. J Inherit Metab Dis. 1994;17(4):383-90.

19. Kluijtmans $L A$, et al. Two novel missense mutations in the cystathionine betasynthase gene in homocystinuric patients. Hum Genet. 1995;96(2):249-50.

20. Shih VE, et al. A missense mutation (I278T) in the cystathionine betasynthase gene prevalent in pyridoxine-responsive homocystinuria and associated with mild clinical phenotype. Am J Hum Genet. 1995;57(1):34-9.

21. Sebastio $G$, et al. The molecular basis of homocystinuria due to cystathionine beta-synthase deficiency in Italian families, and report of four novel mutations. Am J Hum Genet. 1995;56(6):1324-33.

22. Kim CE, et al. Functional modeling of vitamin responsiveness in yeast: a common pyridoxine-responsive cystathionine beta-synthase mutation in homocystinuria. Hum Mol Genet. 1997:6(13):2213-21.

23. Kruger WD, et al. Cystathionine beta-synthase deficiency in Georgia (USA): correlation of clinical and biochemical phenotype with genotype. Hum Mutat. 2003;22(6):434-41.

24. Moat SJ, et al. The molecular basis of cystathionine beta-synthase (CBS) deficiency in UK and US patients with homocystinuria. Hum Mutat. 2004; 23(2):206.

25. Ubbink JB, et al. Vitamin requirements for the treatment of hyperhomocysteinemia in humans. J Nutr. 1994;124(10):1927-33.

26. Bronstrup A, et al. Effects of folic acid and combinations of folic acid and vitamin B-12 on plasma homocysteine concentrations in healthy, young women. Am J Clin Nutr. 1998:68(5):1104-10.

27. Brouwer IA, et al. Low-dose folic acid supplementation decreases plasma homocysteine concentrations: a randomized trial. Am J Clin Nutr. 1999;69(1): 99-104.

28. McKinley MC, et al. Low-dose vitamin B-6 effectively lowers fasting plasma homocysteine in healthy elderly persons who are folate and riboflavin replete. Am J Clin Nutr. 2001:73(4):759-64.

29. Craig SAS. Betaine in human nutrition. Am J Clin Nutr. 2004;80(3):539-49.

30. Sunden SL, et al. Betaine-homocysteine methyltransferase expression in porcine and human tissues and chromosomal localization of the human gene. Arch Biochem Biophys. 1997;345(1):171-4.

31. Gallagher PM, et al. High frequency (71\%) of cystathionine $\beta$-synthase mutation G307S in Irish homocystinuria patients. Hum Mutat. 1995;6(2): 177-80.

32. Gupta S, et al. Mouse modeling and structural analysis of the p.G307S mutation in human cystathionine beta-synthase (CBS) reveal effects on CBS activity but not stability. J Biol Chem. 2018;293(36):13921-31.

33. Wilcken DEL, et al. Homocystinuria - the effects of Betaine in the treatment of patients not responsive to pyridoxine. N Engl J Med. 1983; 309(8):448-53.

34. Nikolic Turnic TR, et al. Efficiency of atorvastatin and simvastatin in improving cardiac function during the different degrees of hyperhomocysteinemia. Can J Physiol Pharmacol. 2018;96(10):1040-9. 
35. Bosze Z, Houdebine LM. Application of rabbits in biomedical research: a review. World Rabbit Sci. 2006;14(1):1-14.

36. Liu H-R, et al. Low cholesteryl ester transfer protein and phospholipid transfer protein activities are the factors making tree shrew and beijing duck resistant to atherosclerosis. Lipids Health Dis. 2010;9.

37. Liu M, Bagdade JD, Subbaiah PV. Specificity of lecithin: cholesterol acyltransferase and atherogenic risk: comparative studies on the plasma composition and in vitro synthesis of cholesteryl esters in 14 vertebrate species. J Lipid Res. 1995;36(8):1813-24.

38. Brown MS, Goldstein JL. A receptor-mediated pathway for cholesterol homeostasis. Science (New York, N.Y.). 1986;232(4746):34-47.

39. Ishibashi $\mathrm{S}$, et al. Hypercholesterolemia in low density lipoprotein receptor knockout mice and its reversal by adenovirus-mediated gene delivery. J Clin Invest. 1993;92(2):883-93.

40. Fan JL, Challah M, Watanabe T. Transgenic rabbit models for biomedical research: current status, basic methods and future perspectives. Pathol Int. 1999;49(7):583-94

41. Lu R, et al. Spontaneous severe hypercholesterolemia and atherosclerosis lesions in rabbits with deficiency of low-density lipoprotein receptor (LDLR) on exon 7. EBioMedicine. 2018;36:29-38.

42. Zhang T, et al. 'Double-muscling' and pelvic tilt phenomena in rabbits with the cystine-knot motif deficiency of myostatin on exon 3. Biosci Rep. 2019; 39(5).

43. Selhub J. The many facets of hyperhomocysteinemia: Studies from the Framingham cohorts. J Nutri. 2006;136(6):1726s-30s.

44. Peng HY, et al. Elevated homocysteine levels and risk of cardiovascular and all-cause mortality: a meta-analysis of prospective studies. J Zhejiang Univ Sci B. 2015;16(1):78-86.

45. Yeh YC, et al. Association of homocysteine level and vascular burden and cognitive function in middle-aged and older adults with chronic kidney disease. Int J Geriatr Psychiatry. 2016;31(7):723-30.

46. Singh LR, et al. Chemical chaperone rescue of mutant human cystathionine ß-synthase. Mol Genet Metabol. 2007;91(4):335-42.

47. Melenovska $P$, et al. Chaperone therapy for homocystinuria: the rescue of CBS mutations by heme arginate. J Inherit Metab Dis. 2015;38(2):287-94.

48. Kopecka J, et al. Restoring assembly and activity of cystathionine betasynthase mutants by ligands and chemical chaperones. J Inherit Metab Dis. 2011;34(1):39-48.

49. Namekata K, et al. Abnormal lipid metabolism in cystathionine betasynthase-deficient mice, an animal model for hyperhomocysteinemia. J Biol Chem. 2004;279(51):52961-9.

50. Watanabe $\mathrm{M}$, et al. Mice deficient in Cystathionine $\beta$-synthase: animal models for mild and severe Homocyst(e)inemia. Proc Natl Acad Sci U S A. 1995;92(5):1585-9.

51. Hirche F, et al. Methionine-induced elevation of plasma homocysteine concentration is associated with an increase of plasma cholesterol in adult rats. Ann Nutr Metab. 2006;50(2):139-46.

52. Baszczuk A, et al. Hyperhomocysteinemia, lipid and lipoprotein disturbances in patients with primary hypertension. Advanc Med Sci. 2014;59(1):68-73.

53. Momin M, et al. Relationship between plasma homocysteine level and lipid profiles in a community-based Chinese population. Lipids Health Dis. 2017;16.

54. Yuan $X$, et al. Associations of homocysteine status and homocysteine metabolism enzyme polymorphisms with hypertension and dyslipidemia in a Chinese hypertensive population. Clin Exp Hypertens. 2019:1-9.

55. Jokinen E. Obesity and cardiovascular disease. Minerva Pediatr. 2015;67(1): 25-32.

56. Barak AJ, Beckenhauer HC, Tuma DJ. Betaine, ethanol, and the liver: a review. Alcohol. 1996;13(4):395-8.

57. Melse-Boonstra A, et al. Betaine concentration as a determinant of fasting total homocysteine concentrations and the effect of folic acid supplementation on betaine concentrations. Am J Clin Nutr. 2005;81(6): $1378-82$.

58. Best $\mathrm{CH}$, Channon $\mathrm{HJ}$. The action of choline and other substances in the prevention and cure of fatty livers. Biochem J. 1935;29(12):2651-8.

59. Morrison LM. Results of betaine treatment of atherosclerosis. Am J Dig Dis. 1952;19(12):381-4.

60. Soler J, et al. Plafibride treatment of hyperlipidemias type II and IV. Med Clin (Barc). 1984;82(19):840-2.

61. Maclean KN, et al. A novel transgenic mouse model of CBS-deficient homocystinuria does not incur hepatic steatosis or fibrosis and exhibits a hypercoagulative phenotype that is ameliorated by betaine treatment. Mol Genet Metab. 2010;101(2-3):153-62.

62. Fan J, Watanabe T. Cholesterol-fed and transgenic rabbit models for the study of atherosclerosis. J Atheroscler Thromb. 2000;7(1):26-32.

63. Salahi $P$, et al. Betaine: a promising micronutrient in diet intervention for ameliorating maternal blood biochemical alterations in gestational diabetes mellitus. Int J Pept Res Ther. 2019;26(2):1177-84.

64. Greeve J, et al. Apolipoprotein B mRNA editing in 12 different mammalian species: hepatic expression is reflected in low concentrations of apoBcontaining plasma lipoproteins. J Lipid Res. 1993;34(8):1367-83.

65. Bosze $Z$, et al. The transgenic rabbit as model for human diseases and as a source of biologically active recombinant proteins. Transgenic Res. 2003; 12(5):541-53.

\section{Publisher's Note}

Springer Nature remains neutral with regard to jurisdictional claims in published maps and institutional affiliations.

\section{Ready to submit your research? Choose BMC and benefit from:}

- fast, convenient online submission

- thorough peer review by experienced researchers in your field

- rapid publication on acceptance

- support for research data, including large and complex data types

- gold Open Access which fosters wider collaboration and increased citations

- maximum visibility for your research: over $100 \mathrm{M}$ website views per year

At BMC, research is always in progress.

Learn more biomedcentral.com/submissions 